\title{
EXPLOITATION OF THE ADDITIVE COMPONENT OF THE POLARIMETRIC NOISE MODEL FOR SPECKLE FILTERING
}

\author{
Samuel Foucher, Grégory Farage* \\ Computer Research Institute of Montreal \\ Vision Team \\ Montreal (Qc), Canada (sfoucher@crim.ca)
}

\author{
Universitat Politécnica de Catalunya (UPC) \\ Signal Theory and Telecommunications Department (TSC) \\ Barcelona, Spain (carlos.lopez@tsc.upc.edu)
}

\begin{abstract}
Ratio filters for speckle noise reduction in SAR imagery are recursive filters where the image structure is iteratively recovered from an initial oversmoothed image. We show that the MBPolSAR filter could be interepreted as a ratio filter applied to the off-diagonal terms of the covariance/coherency matix. From this observation, we propose a new polarimetric ratio filter allowing us to recover the image structure from all the terms of the covariance matrix. In addition, we briefly look at how the additive noise component could also be exploited for the image structure extraction. Filtering results on both simulated and real PolSAR images are shown.
\end{abstract}

Index Terms - PolSAR, Speckle, Filtering, Wavelet

\section{INTRODUCTION}

SAR Polarimetry (PolSAR) has been demonstrated to be an important diversity source for the extraction of bio- and geophysical parameters to characterize the Earth's surface. The sensitivity of PolSAR data to the geometry of the observed targets, together with the sensitivity to different electromagnetic properties, specially permittivity, makes this type of data valuable for thematic classification and for the quantitative extraction of surface parameters such as humidity or roughness. Nevertheless, and due to the coherent nature of SAR systems, PolSAR data are affected by speckle. Under the hypothesis of Gaussian scattering, valid for stationary data, the most important radar observables for PolSAR data are the covariance or the coherency matrices, that need to be estimated from data in order to reduce, or even to eliminate, the speckle effects.

PolSAR speckle noise filtering is still today an unsolved and challenging problem for the operational use of PolSAR data for both, qualitative and quantitative applications [1]. PolSAR filtering algorithms must adapt to different properties of the signal in order to avoid the loss information. First of

\footnotetext{
${ }^{*}$ This work has been supported in part by the NSERC of Canada (Discovery Grant) and the MDEIE of the "Gouvernement du Québec"

${ }^{\dagger}$ This work has been partially funded by the Ramon-y-Cajal program and the I+D project TEC2007-65690/TCM
}

all, PolSAR filtering needs to adapt to the nature of the targets being observed, that is, point or distributed scatterers. Basically, this necessity represents the need to maintain the spatial resolution and the spatial details, as addressed in [2,3]. Additionally, PolSAR speckle filters must also adapt to the nature of the PolSAR data being filtered, as proved in [4].

The adaptability to the data morphology is obtained through a detailed analysis of the neighboring region of the pixel being processed. Some examples in the literature are $[2,3]$. The second level of adaptability is obtained through an exploitation of the data probability density function (pdf). In the case of PolSAR data being distributed according to the zero-mean, complex Gaussian pdf, it was shown that speckle noise results from the combination of multiplicative and additive noise sources [5].

The objective of the work detailed in this paper is twofold. The first part addresses an in-depth exploitation of the multiplicative-additive speckle noise model for PolSAR data. As it has been shown in different contributions, the filtering process of the different noise sources suffers from several inconsistencies due to a insufficient accuracy of the proposed PolSAR speckle noise model to describe the data or due to an insufficient adaptability to the data morphology. In order to reduce these drawbacks, an iterative scheme reprocessing the multiplicative, as well as the additive noise components, is considered in order to retrieve data details that should not have been removed from the original signal. In addition, the filtering of the speckle noise components is performed through a Wavelet scheme in order to recover the image structure and maintain the spatial resolution.

\section{POLSAR DATA FILTERING}

\subsection{The Ratio Filter for Single Band SAR Images}

The Ratio filter for the filtering of single polarization SAR images has been proposed by Bijaoui et al. [6]. This approach directly exploits the multiplicative nature of speckle noise on power images in order to iteratively refine the estimation of the image structure. The method is shown on Algorithm 1. First, a coarse filtering of the image is performed by a Low- 
Pass filter (LP). Then, the image ratio between the original image and the denoised image is formed. Since the denoised images has lost most of the image details such as point target, edges, etc., the details will show up in the ratio image within the background noise, i.e., speckle. A similar approach to study the noise signal obtained after filtering has been proposed in [7]. In [6] the recovery of the image details is performed with the help of a wavelet transform. This process is iterated until no changes are observed in the filtered image.

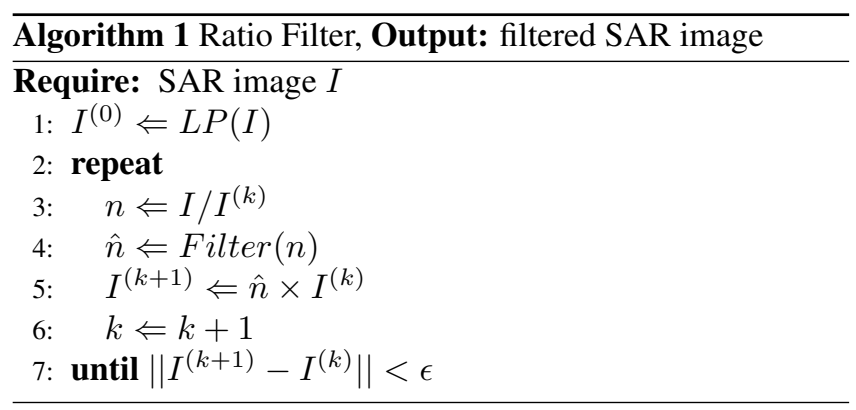

\subsection{The MBPolSAR Filter}

The polarimetric noise model established by López-Martínez and Fabregas [5] leads to the following decomposition for the covariance matrix $\mathbf{Z}$ under the hypothesis of PolSAR data being characterized by the zero-mean, complex Gaussian pdf

$$
\mathbf{Z}=\mathbf{C}+\mathbf{N}_{m}+\mathbf{N}_{a} \text {. }
$$

In particular, all the elements of the covariance matrix may be expressed according to

$S_{p} S_{q}^{H}=\psi|\rho| e^{j \phi_{x}}+\psi \bar{z}_{n} N_{c}\left(n_{m}-1\right) e^{j \phi_{x}}+\psi\left(n_{a r}+j n_{a i}\right)$.

Two noise terms are present, which final contribution is determined basically by the complex coherence value $\rho=|\rho| e^{j \phi_{x}}$. Strong coherence values will imply that the multiplicative noise, $n_{m}$ will be dominant, whereas low coherence values will see the complex additive noise $n_{a r}+j n_{a i}$ prevail. The polarimetric phase determines also the contribution of the multiplicative speckle noise component to the real and imaginary parts of the filtered Hermitian product, in such a way that even in high coherence cases, the additive speckle noise component may dominate. According the the previous PolSAR speckle noise model, the MBPolSAR filter [4], described on Algorithm 2, was developed and validated. The approach presented in [4] took into account the filtering of the multiplicative and the additive speckle noise component. Basically, and as indicated on Algorithm 2, the algorithm reduces first the additive speckle noise component by synthesizing the first additive term of (2) and then, in an iterative way, removes the multiplicative speckle noise component.

It is worth to notice that the original implementation of this filter did not include any adaptation to the image morphology. In the next section, it shall be shown that the MBPol-

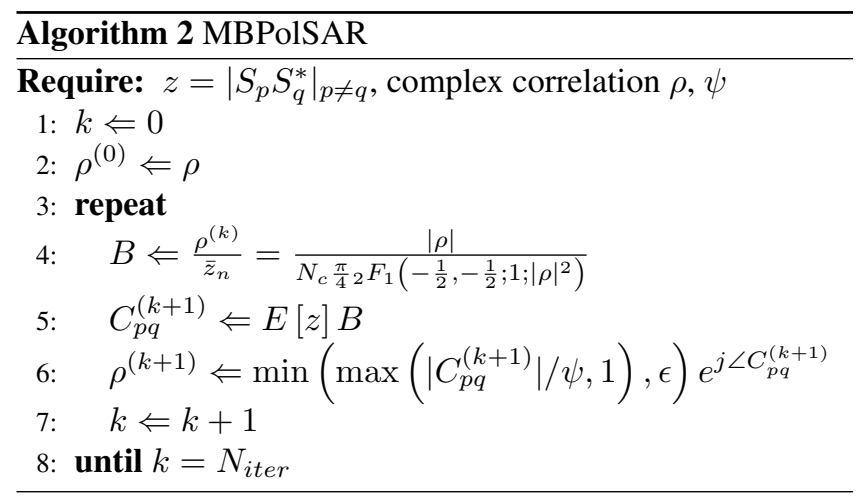

SAR filter may be understood as an extension of the ratio filter.

\subsection{Extension of the Ratio Filter to PolSAR Data}

The averaging operator of line 5 on Algorithm 2 may be rewritten as follows

$$
\hat{C}_{p q}=E[z] B=E\left[n_{m}\right] \psi \rho
$$

where $n_{m}$ is a stationary multiplicative speckle noise component, that is, $E\left[n_{m}\right]=1, \operatorname{var}\left[n_{m}\right]=1$ for single look images. The previous expression may be seen as an iterative scheme similar to line 5 in the ratio filter

$$
\hat{C}_{p q}^{(k+1)}=E\left[n_{m}^{(k)}\right] \hat{C}_{p q}^{(k)}
$$

Therefore, the filtering process of the multiplicative speckle noise component may be performed through a direct extension of the ratio filter, see Algorithm 1, to the off-diagonal elements of the covariance matrix by simply replacing the line 5 by equation (4) above. In addition, it is also important to recover the image structure eventually while smoothing the speckle noise, since as indicated, this was not the purpose of the original implementation of the MBPolInSAR filter. In order to improve this original implementation, different filtering strategies may be addressed. In the ratio filter a wavelet approach was considered, where a simple threshold of the wavelet coefficients was applied [6]. In [9], an entropy based threshold was also proposed. Here, weighting functions $W_{p q}$ are computed for each wavelet band of level $l$ based on a local Wiener filter $w=\max \left(\left(v \hat{a} r^{[l]}-S_{2}^{[l]}\right) / v \hat{a} r^{[l]}, 0\right)$, where $v \hat{a} r^{[l]}$ is the local wavelet coefficient variance and $S_{2}^{[l]}$ is the equivalent power gain similar to [1]. However, in order to preserve the polarimetric information, the same amount of filtering must be applied when filtering all the elements of the sample covariance matrix [4]. In order to achieve this, we compute an average function $W=\frac{1}{6} \sum_{p, q} W_{p q}^{(k)}$ representing the image structures to be recovered. Noise components $n_{p q}^{(k)}$ are then filtered using this function producing residual signals $\hat{n}_{p q}^{(k)}$. In order to preserve the covariance matrix correlation structure, we apply the filtering correction step using 
the average correction signal $\frac{1}{6} \sum_{p, q} \hat{n}_{p q}^{(k)}$ on all the polarimetric channels. The algorithm overview is shown on the Algorithm 3 below.

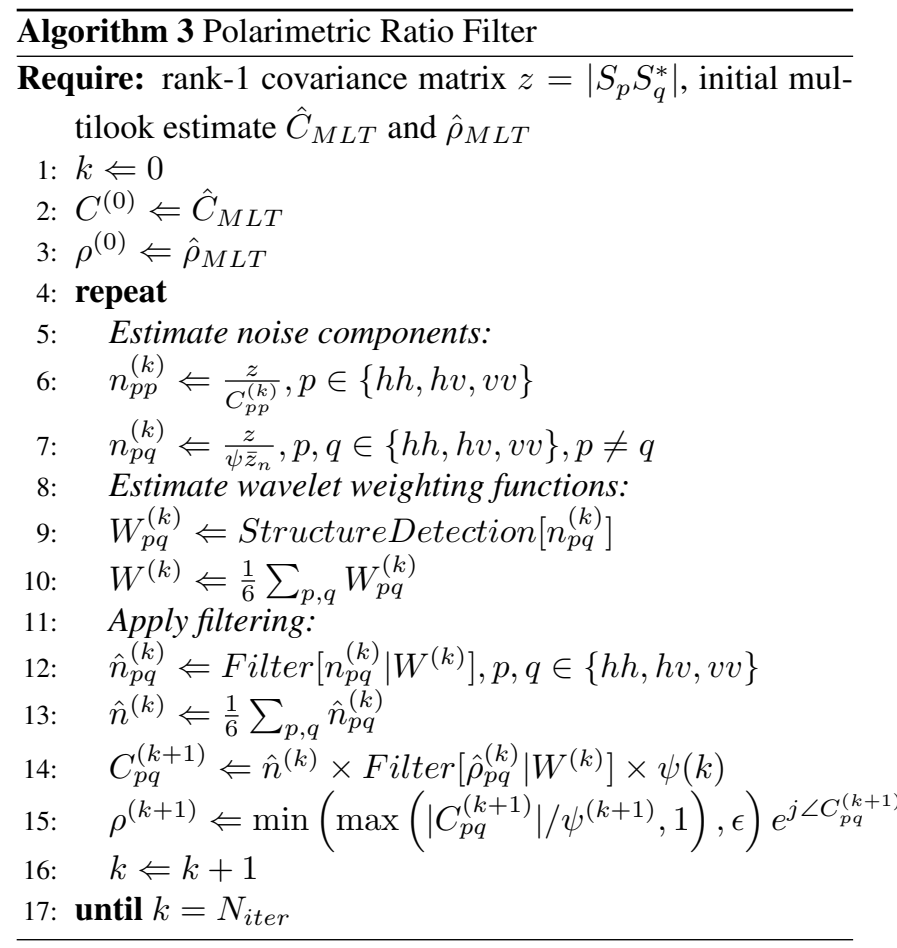

\subsection{A Look at the Additive Noise Component}

The additive component in Equation (2) is a zero noise complex noise with a variance dependent on the coherence value $\sigma_{n_{a r}}^{2}=\sigma_{n_{a i}}^{2}=\frac{1}{2}\left(1-|\rho|^{2}\right)^{1.32}$ [5]. Consequently, exploiting this signal for extracting the image structure is much more challenging and will depend also on the phase signal. The residual noise once the multiplicative noise is removed is the following

$$
\mathbf{a}=\mathbf{n}_{\mathbf{a}}+|\rho| e^{j \phi_{x}}=\frac{S_{p} S_{q}^{H}-\psi \bar{z}_{n} N_{c}\left(n_{m}-1\right) e^{j \phi_{x}}}{\psi} .
$$

Theoretically, we should be able to estimate the polarimetric signal from the mean $E\{\mathbf{a}\}=|\rho| e^{j \phi_{x}}$. The computation of the weight functions need to be modified in order to take into account the non-stationary noise

$$
w=\max \left(\frac{v \hat{a} r^{[l]}-S_{2}^{[l]} \hat{\sigma}_{a}^{2}}{v \hat{a} r^{[l]}}, 0\right)
$$

where $\hat{\sigma}_{a}^{2}=\frac{1}{2}\left(1-|\hat{\rho}|^{2}\right)^{1.32}$.

\section{RESULTS AND EVALUATION}

A simple simulated single-look PolSAR image showing a step edge with two different scattering classes, that in this case correspond to low entropy $(H / A / \bar{\alpha}=0.22 / 0.44 / 51 \mathrm{deg})$ and high entropy $(H / A / \bar{\alpha}=0.92 / 0.12 / 53.8 \mathrm{deg})$. The proposed filter is applied considering 5 iterations. A Stationary Wavelet Transform (SWT) with 3 levels and a short symmetric biorthogonal wavelet is used as the Filter function. The filtering result is shown on Figure 1.

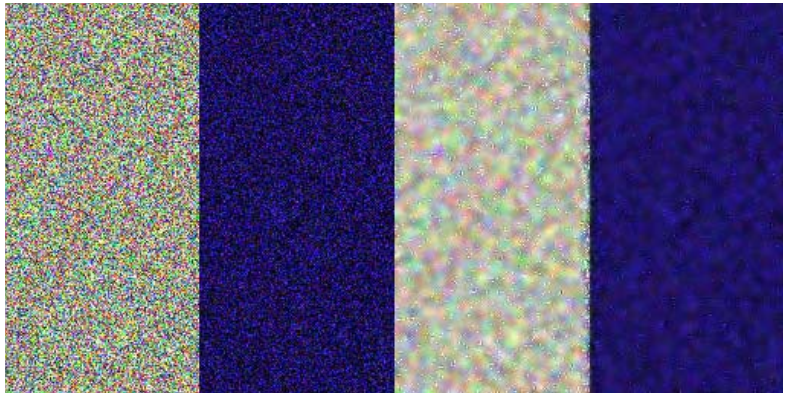

Fig. 1. Original PolSAR image (left) and filtering result with 5 iterations (right). Pauli color coding (HH$\mathrm{VV}, \mathrm{HV}, \mathrm{HH}+\mathrm{VV})$.

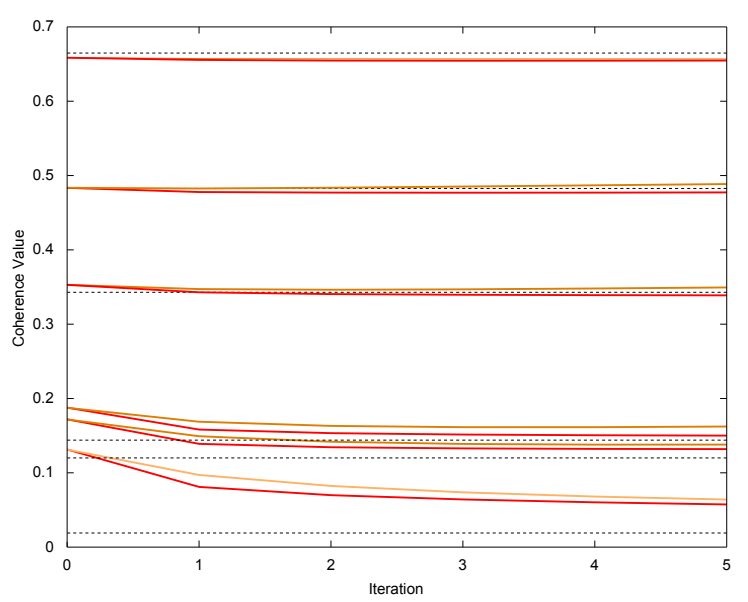

Fig. 2. Coherence values for the simulated image (Figure 1) with asymptotic values (dotted lines), estimates from Algorithm 2 (orange line) and from Algorithm 3 (thick red line) as a function of the iteration. Iteration 0 is the original multilook value $(7 \times 7)$.

As it can be sees on Figure 1, the proposed filter results into a good preservation of local details corresponding to the line separation between both scattering classes. The maintenance of the polarimetric information is presented in Figure 2, where we can observe that the actual coherence values are correctly retrieved. As the filter considers specifically the additive part of speckle, improved estimations of low coherence are obtained. For comparison, we present also the coherence values retrieved by the Algorithm 3, where it may be 
observed that a larger bias reduction is obtained for low coherence values mainly because mixture between polarimetric signatures along the boundary was mitigated. For the proposed method, the relative bias in $\%$ of the asymptotic value is $(H / A / \bar{\alpha})=(0.9 /-22.1 /-4.1) \%$ for the high entropy target and $(H / A / \bar{\alpha})=(-2.1 /-0.7 /-0.1) \%$ for the other one. For the boxcar $7 \times 7$, we get $(H / A / \bar{\alpha})=(2.4 /-60.0 /-12.2) \%$ for the high entropy target and $(H / A / \bar{\alpha})=(0.0 /-3.7 /-$ $0.2) \%$ respectively.

In order to study the performances of the proposed filtering approach, it is also applied to an L-band, $400 \times 400$-pixel E-SAR image in the area of Oberpfaffenhofen near Munich, Germany. These data present a spatial resolution of $3 m \times 3 m$, see Figure 3. This image presents strong point targets which can create strong artefacts with the wavelet transform. Therefore, we first form a mask of strong targets by thresholding the span image above the 98th percentile value. Corresponding values in the PolSAR image are then replaced by low span values in the immediate vicinity of the target before filtering. The Cloude-Pottier parameters are shown on Figure 4 where we can observed that the polarimetric information si somewhat to the multilook filter.

\section{DISCUSSION AND CONCLUSIONS}

We proposed a new approach for PolSAR image filtering that combines the iterative approach of the MBPolSAR filter and the Ratio filter. Significant image structures are progressively extracted from the noise images based on a wavelet transform. One advantage of the method is that it exploits information from all the terms of the covariance matrix. In addition, we keep the iterative improvement of the coherence value estimates that is observed with the original MBPolSAR filter. Many improvements are possible, in particular in the way we extract the image structure and the way we merge individual weighting functions. Also, establishing a good strategy for the preservation of strong point targets is still an open issue with this kind of approach.

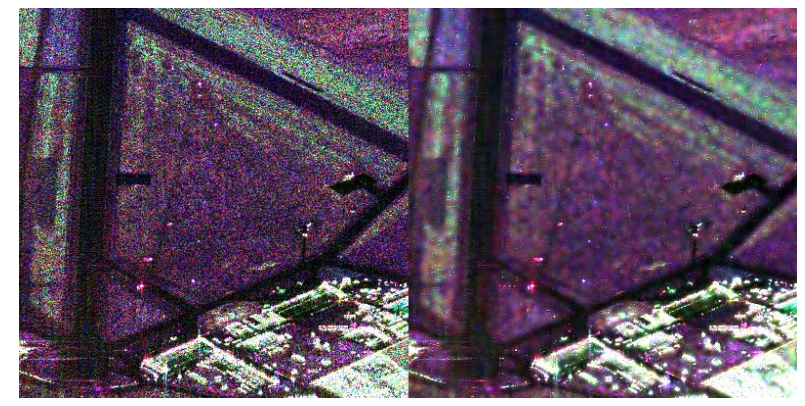

Fig. 3. Original PolSAR image (left) and filtering result with 5 iterations (right). (HH-VV,HV,HH+VV).

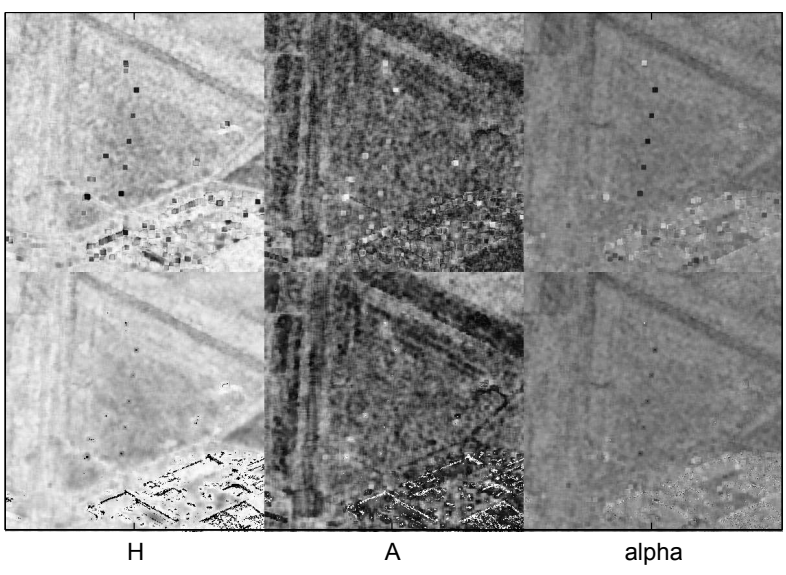

Fig. 4. Cloude-Pottier parameters for the $7 x 7$ boxcar filter (top row) and with the proposed method (bottom row).

\section{References}

[1] S. Foucher and C. López-Martínez, "An evaluation of polsar speckle filters," IGARSS'09, 2009.

[2] J.S. Lee, M.R. Grunes, and G. De Grandi, "Polarimetric sar speckle filtering and its implication for classification," IEEE Trans. Geosci. Remote Sens., vol. 37, no. 5, pp. 2363-2373, 1999.

[3] G. Vasile, E. Trouve, J.S. Lee, and V. Buzuloiu, "Intensitydriven adaptive-neighborhood technique for polarimetric and interferometric sar parameters estimation," IEEE Trans. Geosci. Remote Sens., vol. 44, no. 8, pp. 1609, 2006.

[4] C. López-Martínez and X. Fabregas, "Model-based polarimetric sar speckle filter," IEEE Trans. Geosci. Remote Sens., vol. 46, no. 11, pp. 3894-3907, 2008.

[5] C. López-Martínez and X. Fabregas, "Polarimetric sar speckle noise model," IEEE Trans. Geosci. Remote Sens., vol. 41, no. 10, pp. 2232-2242, 2003.

[6] Fjotoft R. Marthon P. Hervet, E. and A. Lopes, "Comparison of wavelet-based and statistical speckle filters," SPIE, 1998, vol. 3497.

[7] R. Touzi, "A review of speckle filtering in the context of estimation theory," IEEE Trans. Geosci. Remote Sens., vol. 40, no. 11, pp. 2392-2404, 2002.

[8] D. Blacknell R. J. A. Tough and S. Quegan, "A statistical description of polarimetric and interferometric synthetic aperture radar data," Proceedings: Mathematical and Physical Sciences, vol. 449, no. 1937, pp. 567-589, 1995.

[9] G. Farage, S. Foucher, G. Benie, and C. López-Martínez, "Turbo speckle filtering applied on polsar images," IGARSS, 2008, pp. 2648-2652. 